\title{
Development of a clinical decision support system for living kidney donor assessment based on national guidelines
}

Simon R Knight ${ }^{1,2,3}$, Khoa N Cao ${ }^{1,4}$, Matthew South ${ }^{5}$, Nicki Hayward ${ }^{3}$, James $P$ Hunter ${ }^{1}$ and John Fox ${ }^{6}$.

${ }^{1}$ Nuffield Department of Surgical Sciences, University of Oxford, Oxford, UK

${ }^{2}$ Centre for Evidence in Transplantation, Royal College of Surgeons of England, London, UK

${ }^{3}$ Oxford Transplant Centre, Churchill Hospital, Oxford, UK

${ }^{4}$ Austin Health, Melbourne, Australia

${ }^{5}$ Oxford Centre for Human Brain Activity, University of Oxford, Oxford, UK

${ }^{6}$ Lincoln College, University of Oxford, Oxford, UK and OpenClinical CIC

\section{Corresponding Author}

Mr Simon R Knight

Senior Clinical Lecturer and Honorary Consultant Transplant Surgeon

Oxford Transplant Centre

Nuffield Department of Surgical Sciences

Churchill Hospital

Old Road

Oxford

OX3 7LE

Tel: +441865 227131

Mob: +44 7909921103

E-mail: simon.knight@nds.ox.ac.uk 


\section{Authorship}

All authors made a significant contribution to the content of this manuscript, as per ICMJE recommendations.

SRK conceived the study idea, participated in study design, provided clinical input to the decision support system (CDSS), coded the CDSS, entered and analysed patient data and wrote the manuscript. KNC participated in study design, coded the clinical decision support system and reviewed the manuscript.

MS participated in study design, developed the openclinical.net platform, advised on the technical development of the clinical decision support system, provided the tools required for data analysis and reviewed the manuscript.

NH provided clinical input to the content of the clinical decision support system and reviewed the manuscript

JPH participated in study design, provided clinical input to the content of the clinical decision support system and reviewed the manuscript

JF developed the openclinical.net concept, participated in study design, advised on the technical development of the clinical decision support system and reviewed the manuscript.

\section{Disclosures}

JF is a shareholder and Chief Scientific Officer of Deontics Ltd., a commercial company that makes use of the technologies described in this paper. He is also a co-founder of OpenClinical CIC.

MS is a co-founder of both Deontics Ltd and OpenClinical CIC.

KNC is a Medical Al advisor for OpenClinical CIC.

SK, JH and NH have no conflicts of interest in relation to this work.

\section{Funding}

This work did not receive funding from any external sources. 


\section{Abbreviations}

AIDS - acquired immune deficiency syndrome

$\mathrm{BMI}$ - body mass index

BTS - British Transplantation Society

CDSS - Clinical Decision Support System

CJD - Creutzfeldt-Jacob disease

COPD - chronic obstructive pulmonary disease

DMSA - dimercaptosuccinic acid

DNA PCR - deoxyribonucleic acid polymerase chain reaction

DVT - deep vein thrombosis

EDTA - Ethylenediaminetetraacetic Acid

EPAT - ELPAT psychological assessment tool

GFR - glomerular filtration rate

HIV - human immunodeficiency virus

HTLV - human T-lymphotropic virus

HRT - hormone replacement therapy

IHD - ischaemic heart disease

LD - living donor

MAP - mean arterial pressure

MDT - Multidisciplinary Team

$\mathrm{MI}$ - myocardial infarct

NTDT - non-transfusion-dependant thalassaemia

OCP - oral contraceptive pill

OGTT - oral glucose tolerance test

PCR - protein:creatinine ratio

PE - pulmonary embolus

TDT - transfusion-dependant thalassaemia 


\section{Abstract}

Background: Live donor nephrectomy is an operation that places the donor at risk of complications without the possibility of medical benefit. Rigorous donor selection and assessment is therefore essential to ensure minimisation of risk and for this reason robust national guidelines exist. Previous studies have demonstrated poor adherence to donor guidelines.

Methods: We developed a clinical decision support system (CDSS), based upon national living donor guidelines, to facilitate the identification of contraindications, additional investigations, special considerations and the decision as to nephrectomy side in potential living donors. The CDSS was then tested with patient data from 45 potential kidney donors.

Results: The CDSS comprises 17 core tasks completed by either patient or nurse, and 17 optional tasks that are triggered by certain patient demographics or conditions. Decision rules were able to identify contraindications, additional investigations, special considerations and predicted operation side in our patient cohort. 17/45 patients went on to donate a kidney, of whom 7 had major contraindications defined in the national guidelines, many of which were not identified by the clinical team. Only $43 \%$ of additional investigations recommended by national guidelines were completed, with the most frequently missed investigations being oral glucose tolerance testing and routine cancer screening.

Conclusions: We have demonstrated the feasibility of turning a complex set of national guidelines into an easy-to-use machine-readable CDSS. Comparison with real-world decisions suggests that use of this CDSS may improve compliance with guidelines and informed consent tailored to individual patient risks. 


\section{Background}

Donation of a kidney by a living donor represents a useful source of organs for transplantation, providing around $30 \%$ of the kidneys transplanted in the UK each year (1). Living donation does, however, present some specific challenges to the transplant team. Donor nephrectomy is a procedure that places the donor at risk of complications with no potential for medical benefit, and therefore brings unique legal, medical and ethical challenges $(2,3)$. As such, rigorous national and international guidelines have been developed that place donor safety and ethical conditions at the forefront of the assessment process. The process of donor assessment and consent is designed to ensure that the procedure is as safe as possible for the donor, and that the risks of nephrectomy are clearly defined for each individual to allow true informed consent to be obtained.

Living donor guidelines combine ethical, legal, medical and psychological considerations that all need to be assessed in the potential donor. These may include contraindications to donation as well as standard and additional investigations required in the assessment of risk. Such guidelines can be complex - the most recent version of the British Transplantation Society guidelines for living donor kidney transplantation are 293 pages in length (4). The complexity of such guidelines can make it difficult for clinical teams to follow guidance or identify all areas of risk for a potential donor. Indeed, a survey of UK transplant centres identified significant variation in practice between centres with many not adhering to established guidelines in terms of donor eligibility or assessment (5). There is also evidence that this difference in adherence translates to variation in the informed consent process (6).

There is some evidence from other areas of medicine that clinical decision support systems (CDSS), utilising digital representations of guidance, can improve the accuracy of decision making and compliance with clinical guidelines. In simple decisions, such as management of elevated cholesterol, such CDSS have demonstrated improved consistency with guideline-recommended treatment whilst reducing the time and effort required to reach treatment decisions (7). More complex decisions and treatment pathways present a greater a challenge due to the larger number of potentially conflicting decision conditions. However, CDSS have been successfully applied to breast cancer multidisciplinary team (MDT) decisions based upon complex guidelines, again demonstrating improved compliance with guidelines when a CDSS is utilised $(8,9)$. Use of such systems may also facilitate identification of patients eligible for enrolment in clinical trials (9) and help patients to make decisions regarding their own care (10).

In the field of transplantation, the use of CDSS has been more limited. A recent systematic review identified ten studies, mainly investigating the role of CDSS in laboratory test requesting, monitoring of laboratory test results and medication administration (11). CDSS were shown to improve protocol compliance and reduce errors, but no improvements in overall clinical state were documented. No studies investigated the use of CDSS in living organ donors, and none attempted to implement decision systems based upon complex sets of clinical guidelines. 
The aim of the present study was to develop a CDSS for the assessment of living kidney donors, incorporating a patient-completed screening questionnaire, nurse assessment and clinical investigations with decisions based upon national guidelines. Retrospective patient data were used to assess the potential impact of implementing such a CDSS in our centre. 


\section{Methods}

\section{Existing Donor Assessment Pathway}

The existing donor assessment pathway at our centre takes three distinct stages and requires the donor to attend the department on at least three separate occasions. The first stage is donor screening, where the potential donor is asked to complete a screening health questionnaire consisting of medical, psychological and social history. A live donor nurse specialist then reviews this questionnaire with the potential donor during an outpatient clinic, identifying any potential contraindications or considerations relevant to donation. Suitable donors then progress to the medical assessment stage, where they are assessed clinically by a surgeon and nephrologist. Investigations including blood tests, urinalysis, ultrasound, CT angiogram and chromium-EDTA glomerular filtration rate (GFR) are completed for all patients. For patients with a $>10 \%$ discrepancy in size between the left and right kidney, split function is assessed by mean of a dimercaptosuccinic acid (DMSA) scan. Once all investigations are complete, potential donors are discussed at an MDT meeting, following which informed consent is obtained during a final clinic visit prior to surgery.

\section{Development of the Clinical Decision Support System}

A data collection tool was designed to be completed online by potential donors, using the openclinical.net platform. This tool aims to replace the existing screening questionnaire, collecting demographic data, medical and social history and psychological screening questionnaires. Each set of questions represents a specific "task" assessing a particular area of medical or social history. Additional tasks are activated in real-time as a result of data entered; for example, female specific history of pregnancy, pre-eclampsia and cervical screening in response to selection of female gender. This minimises question burden by only showing questions relevant to the user on the screen and therefore completing tasks specific to each individual.

Additional modules allow the nurse specialist to validate the patient-entered information and add the results of laboratory and radiological investigations in a structured format. Raw data are processed to calculate useful derived parameters, such as body mass index (BMI), patient-specific GFR thresholds, kidney size discrepancy and clinical scoring systems.

The openclinical.net platform is based on a well-established approach to the design and deployment of clinical decision support systems which facilitates the definition of machine-executable versions of clinical practice guidelines $(12,13)$. Guidelines are converted to a series of "decision candidates" against which entered data or derived values can be compared to identify patient-specific arguments pro and con each candidate and recommend clinical decisions. These recommendations are continuously updated in the background of the application as new data are entered. For the purposes of the current study, a digital 
representation of the British Transplantation Society Guidelines for Living Donor Kidney Transplantation (4 ${ }^{\text {th }}$ Edition, March 2018) was created (4).

\section{Decision categories}

Four categories of decision were defined from the guidelines. "Contraindications" identify major and minor contraindications to live kidney donation (table 1). Major contraindications are defined as those for which the national guidelines actively discourage donation, whilst minor contraindications are those that increase risk but do not necessarily preclude donation in isolation. "Additional investigations" provide recommendations for any investigations over and above the standard donor pathway, such as oral glucose tolerance testing, echocardiogram, cystoscopy or renal biopsy (table 2). "Special considerations" identify any other considerations unique to that potential donor that need to be taken into consideration during the assessment or consent process, such as the need for an interpreter, requirement for pregnancy counselling, cessation of oral contraception or psychiatric referral (table 3). "Donation side" uses available anatomical and functional data to recommend the safest kidney (left or right) for donation, with the default being left-sided donation if no contraindications are identified (table 4).

\section{Assessment of the CDSS}

In order to test the guideline against real decisions made by the local MDT, we retrospectively entered the data of 45 consecutive potential live kidney donors who presented to the Oxford Transplant Centre between January and March 2016. All available patient history and investigations were entered via the online forms, and the recommended decisions generated for each of the four categories were downloaded. Decisions generated by the CDSS were compared to actual clinical decisions made by the MDT using computer software (pfanalyse).

This retrospective analysis of anonymised data with no resulting alteration to patient care is considered as clinical audit by the NHS research and ethics service, and as such specific ethical approval is not required. 


\section{Results}

Development of the CDSS

The screening component of clinical decision support system comprises 17 core tasks that are completed by all donors (Table S1). In addition, a further 17 tasks are only activated in response to entry of specific user data (medical history, age/gender related screening, social history and previous surgery/hospitalisation). The screening survey takes around ten to fifteen minutes to complete in the absence of a complicated medical history.

The nurse review component comprises two tasks; a review of the medical and social history, and the "red-flag" questionnaire from the ELPAT live donor psychological assessment tool (EPAT) to identify specific social, legal or psychological concerns regarding donation (14).

The laboratory and radiological investigations component comprises 3 core tasks to collate results of routine investigations; blood tests and urinalysis, CT angiogram and GFR measurement. A further optional task collects results of split-function assessment where the size discrepancy between the kidneys is greater than $10 \%$.

Once complete, decisions are provided in the four categories defined above: contraindications, further investigation, special considerations and donation side. Example tasks and decision candidates are illustrated in figure 1.

\section{Comparison of decision recommendations with real patient episodes}

Of the 45 consecutive patients presenting to the unit during the 3-month study period, 17 went on to donate a kidney. The reasons for not proceeding in the remaining 28 patients were: decision to proceed with an alternative live donor $(n=16)$, recipient received a cadaveric kidney transplant $(n=2)$, donor deemed medically unfit $(n=7)$, donor deemed psychiatrically unfit $(n=1)$ and donor withdrew from assessment $(n=2)$.

For the 17 patients that went on to donate, the median time from initial presentation to decision to proceed was 132 days (range 56-626 days). The median time from presentation to donation was 204 days (range 79-650 days).

\section{Contraindications}

The CDSS identified 15 major contraindications in 13 potential donors. Of those with major contraindications, seven proceeded to donation. Two patients had a BMI greater than $35 \mathrm{~kg} / \mathrm{m}^{2}$ but were deemed by the clinical team to be fit to donate after specific discussion with the patient as to the 
additional risks posed. Four patients had persistent traces of leucocytes in their urine in the absence of identifiable infection, and no further investigation was undertaken. One patient had a GFR just below the recommended threshold for age. Another had an initial protein:creatinine ratio greater than 50 $\mathrm{mg} / \mathrm{mmol}$, which was lower than $50 \mathrm{mg} / \mathrm{mmol}$ on two repeat samples with a renal biopsy demonstrating thin basement membrane disease.

Of the seven patients deemed medically unfit to donate, major contraindications were identified in three (2 with BMI greater than $35 \mathrm{~kg} / \mathrm{m}^{2}$, one with a history of breast cancer). A further three had contraindications identified on further investigations recommended by the decision tool; one had retroperitoneal fibrosis apparent on CT scan, another had an adrenal nodule requiring investigation, and the final one had significant cardiac disease identified on a myocardial perfusion study. One further patient had a combination of Minor contraindications that were deemed too high risk to proceed. Twenty-four of the 45 patients had minor contraindications to donation. Of the 17 proceeding to donation, nine had minor contraindications. Common minor contraindications included being a current smoker ( $n=11)$, BMI between $30-35 \mathrm{~kg} / \mathrm{m}^{2}(n=7)$, persistent microscopic haematuria $(n=6)$, history of preeclampsia or hypertension $(n=5)$ and history of psychiatric disease or poor social support $(n=4)$.

\section{Additional investigations}

The CDSS recommended additional investigations in 38/45 potential donors. Of the 93 additional investigations recommended, 40 (43\%) were actually requested and completed. The majority of discrepancies related to the recommendation for oral glucose tolerance testing and the requirement for routine cancer screening tests. National guidelines recommend oral glucose tolerance testing in patients with high fasting blood sugar, $\mathrm{BMI}>35 \mathrm{~kg} / \mathrm{m}^{2}$, of high-risk ethnicity or with a family history of diabetes. Oral glucose tolerance test (OGTT) was only completed in $4 / 23$ cases in which it was recommended.

Bowel cancer screening is offered to all patients aged 60-74 every two years in the UK. Of those eligible, only 1 of 16 had undertaken recent bowel cancer screening, and this was not requested prior to donation in the remaining cases. In two patients with a history of renal stones, metabolic screen or 24-hour urine collections were not completed as per national guidelines.

Compliance with other investigations was much higher. Patients with hypertension at presentation are recommended to undergo 24-hour blood pressure monitoring, which was undertaken in 9 of 11 patients in which it was recommended. Requirements for up-to-date cervical smears and mammograms in female patients were adhered to in 8 of 10 patients in whom these were required. For patients with persistent microscopic haematuria ( $n=6)$, cystoscopy was requested for 4 , and biopsy for 3 . 
In two patients, investigations were undertaken that were not recommended by the CDSS. These were a thrombophilia screen and an echocardiogram, both deemed clinically necessary by the assessing anaesthetist.

\section{Special considerations}

The decision tool identified special considerations in 19/45 patients. Referral to a geneticist was recommended in 5 patients due to a history of potentially inheritable kidney disease. Referral was deemed unnecessary by the nephrologist in all cases due to the nature of the history provided and documented, suggesting appropriate management of these patients.

Referral to a psychiatrist was recommended on the basis of clinical history or nurse concern for five patients, all of whom underwent further assessment by a psychologist or psychiatrist. One patient of childbearing age indicated the desire for a future pregnancy, and the need for pregnancy counselling was correctly identified. Five patients were correctly identified as taking either the oral contraceptive pill or hormone replacement therapy that needed to be stopped prior to surgery.

\section{Donation side}

The decision tool recommended a donation side (left or right kidney) if data from a CT angiogram and GFR measurement were provided. Sufficient data were available for a decision for 25 patients; the remaining patients did not proceed far enough through the donation pathway for these investigations to be completed.

The decision tool identified the same donation side as the clinical MDT in 23/25 patients. In two patients, a significant size discrepancy was identified ( $>1 \mathrm{~cm}$ difference between kidneys). National guidelines recommend assessment of split function with a DMSA scan in these cases, but this was not completed, and the larger kidney was donated in both cases. 


\section{Discussion}

This study demonstrates the feasibility of applying a clinical decision support system to a complex, guideline-driven clinical pathway assessing potential live kidney donors. Use of patient- and nurseentered data in this way has the potential to identify contraindications and necessary additional investigations, improving compliance with guidelines and ultimately reducing donor risk whilst allowing true informed consent specific to the individual patient.

We have demonstrated that a CDSS has the potential to identify contraindications and investigations defined by guidelines that may be overlooked by the clinical MDT. This is particularly important given the nature of live kidney donation, where the safety of the potential donor is the overriding consideration. Seven of the 17 patients proceeding to donation in the current study had major contraindications as defined by the national guidelines. Whilst in some of these, adequate consideration was given to the impact of these contraindications and discussion with the patient was documented, there were a number of cases where no patient discussion or MDT consideration was recorded, suggesting that they may not have been identified by the MDT. Whilst guidelines ultimately advise as to best practice, clinical practice that contradicts best available evidence should be carefully considered, documented, discussed as part of the informed consent process and future guidelines revision. A CDSS such as the one developed here will reduce the chance of overlooking specific clinical risks, providing a personalised risk assessment for each individual patient that can be used as an integral part of the informed consent process. Further developed, it may provide an effective feedback loop in advancing guidelines.

Similarly, the CDSS was able to identify a number of additional investigations that were recommended by national guidelines but were not completed. Again, clinical judgement may dictate that in individual cases the risks of further investigation outweigh the need, but the use of such a tool will provide guidelinebased recommendations to ensure that these considerations are not overlooked. In particular, it helps to identify where cancer screening is not up-to-date, especially where algorithms are complicated and difficult to remember.

The finding of poor compliance with national guidelines is not unique to our centre. A previous study demonstrated a great deal of variability in live kidney donor assessment between UK centres in factors such as age and BMI thresholds, exclusion on the basis of medical history, and the use of investigations (5). Practice in many centres disagreed with national guidelines, suggesting a role for a CDSS in highlighting evidence-based practice.

The use of a patient- and nurse-completed CDSS may have additional benefits over and above guideline compliance and patient safety. In the current analysis, median time from presentation to decision to proceed with a donor was 132 days, and to donation was 204 days, with a great deal of variation between patients. Whilst some of this variation was due to recipient factors or use of the Live Donor Kidney Sharing Scheme, in a number of donors prolonged assessment resulted from sequential 
requesting of investigations or clinical review by other services (e.g. psychiatry). Remote entry of data by patients in their home environment, along with earlier identification of necessary investigations, may improve the co-ordination of investigations and reduce the duration of assessment and number of clinic visits required. This may be important, as the time required for clinic visits has been identified as a potential barrier to the donation process, with improvements in donation rates seen with a one-stop clinic model (15). CDSS use may also prevent unsuitable candidates from progressing further down the assessment pathway. All of this has the potential to save patient and nurse time, and ultimately reduce service costs.

There are a number of limitations to the present study. The single-centre nature of our analysis means that we are unable to assess the potential impact of this tool in other transplant centres, where compliance with guidelines may differ. Whilst retrospective analysis of past decisions suggests some room for improvement, we were unable to establish the true impact of a CDSS used in a prospective setting. Previous studies using CDSS for more straightforward clinical decisions have suggested improved compliance with published guidance when the CDSS is used at the point of decision making. A systematic review of studies reporting the use of CDSS in a number of different settings (drug prescribing, reminders, diagnostics and disease management) identified some evidence for improved practitioner compliance, although very few trials documented improved patient outcomes as a result (16). Similar improvements in compliance have been demonstrated in the more complex setting of cancer treatment decisions (8), and in reducing unnecessary investigation in preoperative assessment (17).

Successful use of a CDSS relies on clinicians acting upon the clinical decisions provided by the system. This will only happen if the CDSS correctly reflects clinical practice guidelines, and if clinicians believe in the recommendations produced in such guidelines. Good quality guidelines recommend practice based upon the best available evidence via a robust guideline development process. Recent work has demonstrated that the quality of international guidelines in renal transplantation is variable, with guidelines lacking in robust developmental methodology which may have an impact on their uptake in real clinical practice (18). It is therefore important that clinical decisions do not just rely on guideline-based CDSS, but also take into account local practice and clinical experience as part of the live donor MDT process. However, a CDSS such as the one presented here can be adapted to local protocols and practice, which may reduce the risk of non-compliance with recommendations when evidence is lacking or of poor quality.

The CDSS developed in this study relies in part on data entry by patients, replacing the use of an existing screening questionnaire. Many transplant centres use a paper-based or online screening questionnaire to identify basic demographic, medical and social data and to rapidly screen out unsuitable donors. Use of this data may speed up the assessment process but will still require verification by a healthcare professional to ensure accuracy before a final decision can be made. Completion of the patient tasks in the current CDSS takes less than 15 minutes, making it feasible for the majority of patients. Further 
refinement by populating patient demographics and investigation results directly from the electronic health record will reduce patient and nurse time further and help to prevent transcription errors. The ability to save the form to return to at a later date would also enhance the patient experience. Future incorporation of tools that allow calculation of patient-specific risk of outcomes such as end-stage renal disease may further enhance the ability of a CDSS to assist in patient-tailored informed consent (19). In summary, we have demonstrated the feasibility of turning a complex set of clinical practice guidelines for the assessment of potential live donors into a machine-executable data and decision-making model. This system is able to accurately identify contraindications, investigations and the most suitable procedure based upon a combination of patient- and nurse-entered data and has the potential to improve evidence-based clinical decision making and patient-specific informed consent. Planned future work will develop this into a patient-facing system and assess the potential impact of this tool in the prospective setting, assessing external validity in other transplant centres. 


\section{References}

1. NHS Blood and Transplant. Organ Donation and Transplant Activity report 2016/17 [Internet]. 2017; [cited 2018 Apr 30] Available from: https://nhsbtdbe.blob.core.windows.net/umbraco-assetscorp/4657/activity_report_2016_17.pdf

2. Truog RD. The Ethics of Organ Donation by Living Donors. New England Journal of Medicine 2005; 353: 444 .

3. Tong A, Chapman JR, Wong G, Craig JC. Living kidney donor assessment: challenges, uncertainties and controversies among transplant nephrologists and surgeons. American Journal of Transplantation: Official Journal of the American Society of Transplantation and the American Society of Transplant Surgeons 2013; 13: 2912.

4. British Transplantation Society. Guidelines for Living Donor Kidney Transplantation, Fourth Edition [Internet]. 2018 [cited 2018 May 30] .Available from: https://bts.org.uk/wpcontent/uploads/2018/03/BTS_RA_LDKT_Guidelines_FINAL_12.03.18.pdf

5. Arunachalam C, Garrues M, Biggins F, Woywodt A, Ahmed A. Assessment of living kidney donors and adherence to national live donor guidelines in the UK. Nephrology, Dialysis, Transplantation: Official Publication of the European Dialysis and Transplant Association - European Renal Association 2013; 28: 1952.

6. Thiessen C, Kim YA, Formica R, Bia M, Kulkarni S. Written informed consent for living kidney donors: practices and compliance with CMS and OPTN requirements. American Journal of Transplantation: Official Journal of the American Society of Transplantation and the American Society of Transplant Surgeons 2013; 13: 2713.

7. Scheitel MR, Kessler ME, Shellum JL et al. Effect of a Novel Clinical Decision Support Tool on the Efficiency and Accuracy of Treatment Recommendations for Cholesterol Management. Applied Clinical Informatics 2017; 8: 124.

8. Séroussi B, Bouaud J, Gligorov J, Uzan S. Supporting multidisciplinary staff meetings for guidelinebased breast cancer management: a study with OncoDoc2. AMIA ... Annual Symposium proceedings. AMIA Symposium 2007; : 656.

9. Patkar V, Acosta D, Davidson T, Jones A, Fox J, Keshtgar M. Using computerised decision support to improve compliance of cancer multidisciplinary meetings with evidence-based guidance. BMJ Open 2012; 2: e000439.

10. Miles A, Chronakis I, Fox J, Mayer A. Use of a computerised decision aid (DA) to inform the decision process on adjuvant chemotherapy in patients with stage II colorectal cancer: development and preliminary evaluation. BMJ Open 2017; 7: e012935.

11. Niazkhani Z, Pirnejad H, Rashidi Khazaee P. The impact of health information technology on organ transplant care: A systematic review. International Journal of Medical Informatics 2017; 100: 95.

12. Sutton DR, Fox J. The Syntax and Semantics of the PROforma Guideline Modeling Language. Journal of the American Medical Informatics Association : JAMIA 2003; 10: 433.

13. Fox J. Cognitive systems at the point of care: The CREDO program. Journal of Biomedical Informatics 2017; 68: 83. 
14. Massey EK, Timmerman L, Ismail SY et al. The ELPAT living organ donor Psychosocial Assessment Tool (EPAT): from "what" to "how" of psychosocial screening - a pilot study. Transplant International: Official Journal of the European Society for Organ Transplantation 2018; 31: 56.

15. Graham JM, Courtney AE. The Adoption of a One-Day Donor Assessment Model in a Living Kidney Donor Transplant Program: A Quality Improvement Project. American Journal of Kidney Diseases 2018; 71: 209.

16. Garg AX, Adhikari NKJ, McDonald H et al. Effects of Computerized Clinical Decision Support Systems on Practitioner Performance and Patient Outcomes: A Systematic Review. JAMA 2005; 293: 1223.

17. Sim EY, Tan DJA, Abdullah HR. The use of computerized physician order entry with clinical decision support reduces practice variance in ordering preoperative investigations: A retrospective cohort study. International Journal of Medical Informatics 2017; 108: 29.

18. O'Donoghue K, Reed R, Knight S et al. Critical Appraisal of International Clinical Practice Guidelines in Kidney Transplantation using the Appraisal of Guidelines for Research and Education (AGREE) II Tool: A Systematic Review. Transplantation 2018; In press.

19. Park JS, Ahn HK, Na J et al. Development of a Screening Tool to Predict Chronic Kidney Disease Risk in Post-nephrectomy Living Kidney Donors. Transplantation Proceedings 2018; 


\section{Figures}

Figure 1 - Example tasks and decision candidates from the clinical decision support system. A - A

patient task to be completed by all patients. B - A conditional patient task activated by a response to a previous task. C - Example decision candidates for contraindications to donation. D - Example decision candidates for recommended further investigations. Recommended decision candidates are determined by comparison of patient-specific data with guideline-defined arguments for and against each decision. 


\section{Tables}

Table 1 - Decision rules for major and minor contraindications to donation. Adapted from the British Transplantation Society (BTS) guidelines for living donor kidney transplantation ( $4^{\text {th }}$ edition) (4).

HIV - human immunodeficiency virus; AIDS - acquired immune deficiency syndrome; CID - Creutzfeldt-Jacob disease; BMI - body mass index; HTLV - human T-lymphotropic virus; TDT transfusion-dependant thalassaemia; NTDT - non-transfusion-dependant thalassaemia; PCR - protein:creatinine ratio; GFR-glomerular filtration rate.

\begin{tabular}{|c|c|}
\hline Contraindication & Major/Minor \\
\hline High-risk cancer at any time (breast, lung, melanoma, testicular, myeloma, choriocarcinoma, renal cell carcinoma, sarcoma) & Major \\
\hline History of HIV/AIDS & Major \\
\hline $\mathrm{CJD} /$ prion disease & Major \\
\hline HTLV1/2 & Major \\
\hline BMI greater than $35 \mathrm{~kg} / \mathrm{m}^{2}$ & Major \\
\hline Chronic kidney disease & Major \\
\hline Sickle cell disease & Major \\
\hline Urine PCR $>50 \mathrm{mg} / \mathrm{mmol}$ & Major \\
\hline Persistent pyuria & Major \\
\hline Hypertension requiring 3 or more drugs & Major \\
\hline
\end{tabular}




\begin{tabular}{|c|c|}
\hline GFR below recommended threshold & Major \\
\hline Malignancy $>5$ years ago & Minor \\
\hline Hepatitis & Minor \\
\hline Hypertension & Minor \\
\hline Pre-eclampsia or eclampsia & Minor \\
\hline Change in bowel habit/weight loss & Minor \\
\hline Previous renal stones & Minor \\
\hline Recurrent urinary tract infections/pyelonephritis & Minor \\
\hline Family history of genetic renal disease & Minor \\
\hline Ischaemic heart disease, stent or bypass & Minor \\
\hline Current smoker & Minor \\
\hline Potentially harmful alcohol intake (> 30 units/wk) & Minor \\
\hline Previous natural growth hormone treatment & Minor \\
\hline History of VTE & Minor \\
\hline Previous surgical or anaesthetic complications & Minor \\
\hline Thalassaemia minor & Minor \\
\hline Sickle cell trait & Minor \\
\hline BMI $30-35$ kg/m² & Minor \\
\hline Poor social support & Minor \\
\hline
\end{tabular}




\begin{tabular}{|c|c|}
\hline Significant psychiatric history & Minor \\
\hline Elevated urine $\mathrm{PCR}>15-50 \mathrm{mg} / \mathrm{mmol}$ & Minor \\
\hline Anaemia of undiagnosed cause & Minor \\
\hline Diabetes & Minor \\
\hline High fasting blood glucose (>6.1 mmol/L) & Minor \\
\hline
\end{tabular}


Table 2 - Decision rules for additional investigations required. Adapted from the British Transplantation Society (BTS) guidelines for living donor kidney transplantation ( $4^{\text {th }}$ edition) (4).

DNA PCR - deoxyribonucleic acid polymerase chain reaction; MI - myocardial infarct; IHD - ischaemic heart disease; COPD - chronic obstructive pulmonary disease; MAP - mean arterial pressure; BMI - body mass index; DVT - deep vein thrombosis; PE - pulmonary embolus; DMSA - dimercaptosuccinic acid.

\begin{tabular}{|c|c|}
\hline Investigation & Decision rule \\
\hline Pregnancy test & Female patients who indicate risk of pregnancy or inadequate contraception \\
\hline Echocardiogram & $\begin{array}{l}\text { Patients reporting shortness of breath, ankle swelling, chest pain, history of heart failure, } \\
\text { history of MI/angina/IHD, history of heart murmur or valve disease, previous cardiac } \\
\text { surgery, history of hypertension }\end{array}$ \\
\hline 24-hour tape & Patients reporting palpitations, syncope, arrhythmia or pacemaker \\
\hline Ambulatory blood pressure monitoring & History of hypertension, antihypertensive drug use, MAP > $106 \mathrm{mmHg}$ \\
\hline Cervical spine imaging & History of arthritis or trauma to neck, history of neck pain \\
\hline Hepatitis B and C DNA PCR & History of hepatitis \\
\hline 24-hour urine collection & History of renal stones \\
\hline Lung function tests & Current or ex-smoker, asthma, COPD, interstitial lung disease \\
\hline $\begin{array}{l}\text { Metabolic screen (urine and plasma biochemistry and 24-hour urine collections for calcium, } \\
\text { oxalate, citrate and urate, and early morning pH assessment) }\end{array}$ & $\begin{array}{l}\text { History of renal stones } \\
\text { History of inflammatory bowel disease }\end{array}$ \\
\hline Oral glucose tolerance test & $\begin{array}{l}\text { High risk ethnicity, family history of diabetes, } \mathrm{BMI}>30 \mathrm{~kg} / \mathrm{m}^{2} \text {, history of gestational } \\
\text { diabetes, high fasting blood glucose (>6.1 mmol/L) }\end{array}$ \\
\hline Myocardial perfusion scan & History of chest pain, angina, MI, IHD or cardiac surgery \\
\hline
\end{tabular}




\begin{tabular}{|c|c|}
\hline Thrombophilia screen & History of DVT/PE, family history of blood clotting disorders \\
\hline Thyroid function tests & History of thyroid disease \\
\hline Sleep studies & High or intermediate-risk stop-bang score, Epworth score $>10$ \\
\hline Syphilis serology & Risk of syphilis \\
\hline Colonoscopy or colorectal referral & Blood in the stool, recent weight loss, change in bowel habit, undiagnosed anaemia \\
\hline Mammography & As per national screening guidelines \\
\hline Cervical smear test & As per national screening guidelines \\
\hline Bowel screening & As per national screening guidelines \\
\hline Cystoscopy & Aged $>40$ with microscopic haematuria \\
\hline Renal biopsy & Persistent microscopic haematuria with negative cystoscopy \\
\hline DMSA & $>10 \%$ difference in kidney size \\
\hline Haemoglobinopathy screen & Patients of non-Northern European Heritage \\
\hline Prostate-specific antigen (PSA) & All men over 55 years \\
\hline
\end{tabular}


Table 3 - Decision rules for special considerations. Adapted from the British Transplantation Society (BTS) guidelines for living donor kidney transplantation (4th edition) (4).

OCP - oral contraceptive pill; HRT - hormone replacement therapy; EPAT - ELPAT psychological assessment tool.

\begin{tabular}{|l|l|}
\hline Consideration & Decision rules \\
\hline Interpreter required & Patient indicates need for interpreter \\
\hline Referral to geneticist & Family history of genetic renal disorder \\
\hline Pregnancy counselling & Patient indicates plans for future children \\
\hline Cessation of contraceptive pill/HRT & Patient is currently taking OCP/HRT \\
\hline Referral to psychiatrist & Patient has a history of drug or alcohol abuse or history of psychiatric disorder. \\
& Patient is a non-directed donor. \\
The EPAT screening tool indicates risk of anxiety, depression, psychiatric disorder, poor \\
resilience or poor social support. \\
The nurse identifies risk of any of the above.
\end{tabular}


Table 4 - Decision rules for operation side. Adapted from the British Transplantation Society (BTS) guidelines for living donor kidney transplantation (4 ${ }^{\text {th }}$ edition) (4).

\begin{tabular}{|c|c|}
\hline Decision & Decision rules \\
\hline Left kidney & $\begin{array}{l}\text { Left kidney is }>10 \% \text { smaller than right } \\
\text { Left kidney has }<45 \% \text { split function } \\
\text { Right kidney has more arteries than the left } \\
\text { Right kidney has early arterial branching } \\
\text { Right kidney has more veins than the left } \\
\text { Left kidney has one or more renal stones }\end{array}$ \\
\hline Right kidney & $\begin{array}{l}\text { Right kidney is }>10 \% \text { smaller than right } \\
\text { Right kidney has }<45 \% \text { split function } \\
\text { Left kidney has more arteries than the right } \\
\text { Left kidney has early arterial branching } \\
\text { Left kidney has more veins than the right } \\
\text { Right kidney has one or more renal stones }\end{array}$ \\
\hline
\end{tabular}

